\title{
Modeling a Planar Coupled Microstrip Lines using various Wavelets and Method of Moments
}

\author{
Mohamed Bayjja ${ }^{1}$, Mahmoud Moubadir ${ }^{1}$, Gamil Alsharahi², Mohamed Aghoutane ${ }^{1}$, Naima Amar \\ Touhami $^{1}$ \\ ${ }^{1}$ Laboratory Information Systems and Telecommunications, Department Physics, Faculty of Science, University Abdelmalek \\ Essaâdi, Tetouan, Morocco. \\ ${ }^{2}$ Laboratory Communication Systems, Department Physics, Faculty of Science, University Abdelmalek Essaâdi, Tetouan, \\ Morocco. \\ *corresponding author, E-mail: m.bayjja@gmail.com
}

\begin{abstract}
In this paper, we apply a several wavelets basis functions to the method of moments to modeling the parallel-coupled microstrip lines. The first set of equations is for the shielded microstrip line solved with moment's method and wavelets. The Green's function is obtained from the theory of images. The second set are for the parallel-coupled microstrip lines operating in the TEM mode or when the analysis can be based on quasi-static approximation, the properties of coupled lines can be determined from the self- and mutual inductances and capacitances for the lines. To demonstrate the effectiveness and accuracy of the proposed technique, numerical results of even- and odd-mode characteristic impedances, coupling coefficient, percentage sparsity achieved in the impedance matrix, the CPU Time to reverse impedance matrix, and error relative for Daubechies, Coiflets, Biorthogonal and Symlets wavelets are presented. Numerical results are in good agreement with those in previous publications.
\end{abstract}

\section{Introduction}

Since the first carried out research to nowadays, transmission line-based microwave devices are largely used in telecommunication systems to constantly improve the overall performances by reducing the size, weight, and cost. The parallel-coupled microstrip lines are utilized extensively as basic elements for directional couplers, filters, phase shifters and a variety of others useful circuits $[1,2]$. Because of the coupling of electromagnetic fields, a pair of coupled lines can support two different modes of propagation. These modes have different characteristic impedances. The velocity of propagation of these two modes is equal when the lines are embedded in a homogeneous dielectric medium $[3,4]$.

Wavelet theory is a relevant continuously emerging area in mathematical research. It has been applied to a wide range of engineering disciplines and received considerable attention on computational electromagnetics, particularly in solving integral equations $[5,6]$.

Moment method is an accurate numerical method, but needs huge computational time and space memory, for this reason it cannot be applied to structures larger than a few wavelengths. Although several fast algorithms have been used to reduce the computational complexity and memory requirement, but need more powerful machine to be implemented. In contrast, Wavelet-Based Moment method which can be implemented easily in personal computer. By using the wavelets expansions, the basis and testing functions are chosen based for MoM. Owing to the orthogonality, vanishing moments, and multiresolution analysis of wavelets, a very sparse moment matrix is obtained. The interest in the sparse matrix not only from the reduction of the memory space (one does not store the zeros) but also from the reduction of the number of operations (one will not perform the operations on the zeros), reduction in computation time $[7,8]$.

The paper is organized as follows. Section 2 is devoted to microstrip line and coupled Microstrip formulation of the integral equation, using Green's function. In Section 3, the coupled mode approach is used to characterize the coupled line by the characteristic impedances for the two modes. These two modes are obtained by considering the effect of the self- and the mutual inductances and capacitances on the modes in the individual uncoupled lines. Numerical results and discussion are also presented to compare several wavelet bases for their merits in solving the problem in hand.

\section{Analysis of Microstrip Line and coupled Microstrip Lines.}

\subsection{Analyses of Microstrip Line}

In the analysis, we consider a shielded microstrip line with the cross-section shown in Figure $1[9,10]$. We assume that the metal and dielectric losses in this line are negligible and the propagation mode in direction $\mathrm{Oz}$ is almost TEM. The function $G$ satisfies the boundary and the interface conditions of the microstrip configuration but not the source condition. Using Green's function, an integral equation of the following form can be formulated and solved to determine the charge distribution [10]: 


$$
V(x, y 0)=V(x)=\int_{\frac{a-w}{2}}^{\frac{a+w}{2}} G(x, x 0) \rho(x 0) d x 0
$$

Where $\mathrm{V}$ and $\rho$ are the potential and the charge distributions, respectively.

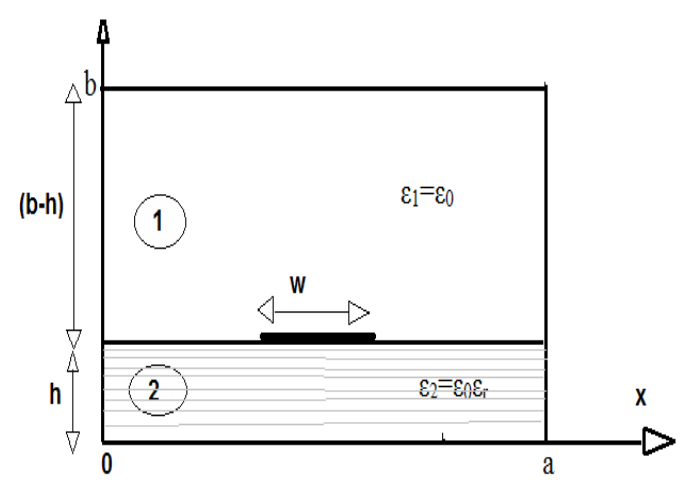

Figure1: Shielded microstrip line geometry [10].

Green's function $G$ for the microstrip configuration is obtained from the theory of images for a charge placed in front of a dielectric-air interface and has been described by Silvester [3]. The method of images is illustrated in Figure 2 . In this figure, the partial image coefficient $\mathrm{K}$ is given by $[11,12]$

$$
K=\frac{\left(1-\varepsilon_{r}\right)}{\left(1+\varepsilon_{r}\right)}
$$

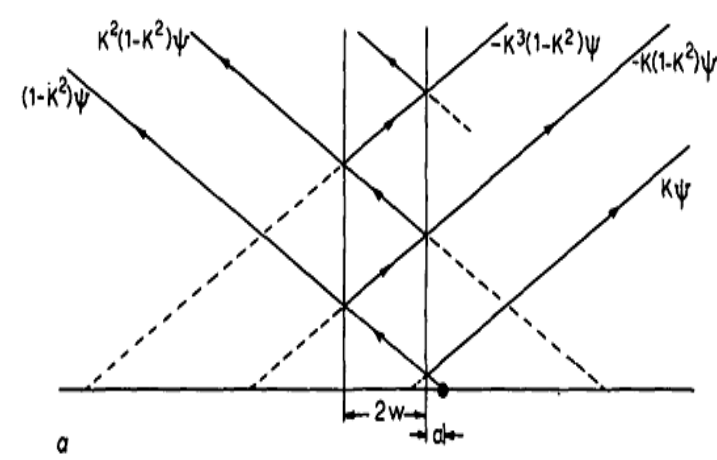

Figure 2: Multiple images of a line charge in front of a dielectric slab

The Green's function G for the configuration as shown in Fig. 1 may be written as [3]:

$$
\begin{aligned}
G(h, x)=\frac{1}{2 \pi\left(\varepsilon_{r}+1\right) \varepsilon_{0}}\left\{\sum _ { n = 1 } ^ { \infty } K ^ { n - 1 } \cdot \operatorname { l n } \left[\left\{( 4 n ^ { 2 } + ( \frac { x - x 0 } { h } ) ^ { 2 } ) \left(4 n^{2}\right.\right.\right.\right. \\
\left.\left.+\left(\frac{x+x 0}{h}\right)^{2}\right)\right\} \\
\\
/\left\{( 4 ( n - 1 ) ^ { 2 } + ( \frac { x - x 0 } { h } ) ^ { 2 } ) \left(4(n-1)^{2}\right.\right. \\
\left.\left.\left.\left.+\left(\frac{x+x 0}{h}\right)^{2}\right)\right\}\right]\right\}
\end{aligned}
$$

\subsection{Analyses of coupled Microstrip Lines}

A "coupled line" configuration consists of two transmission lines placed parallel to each other and in close proximity as shown in Figure 3. In such a configuration there is a continuous coupling between the electromagnetic fields of the two lines [3].

For the lines operating in the TEM mode or when the analysis can be based on quasi-static approximation, the properties of coupled lines can be determined from the selfand mutual inductances and capacitances for the lines. In the case of lines operating in the non-TEM mode (for example, coupled slotlines), a full wave analysis is needed for the two modes of propagation.

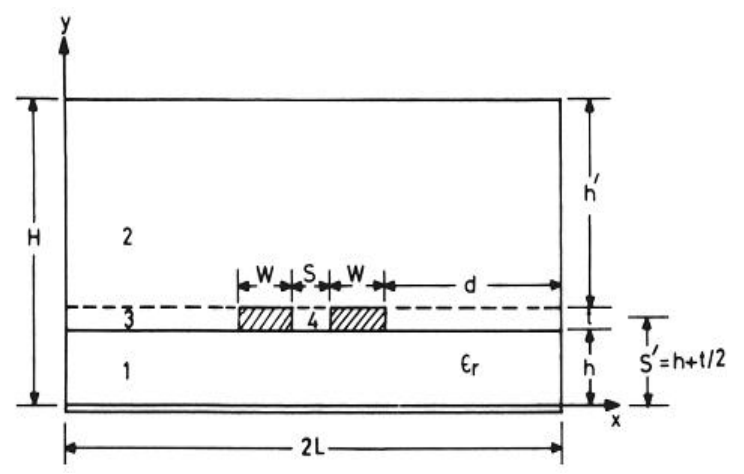

Figure 3: Symmetric coupled microstrip lines in an enclosure [3].

The coupled mode analysis for coupled lines with unequal impedances, shown in Figure 4, is described next

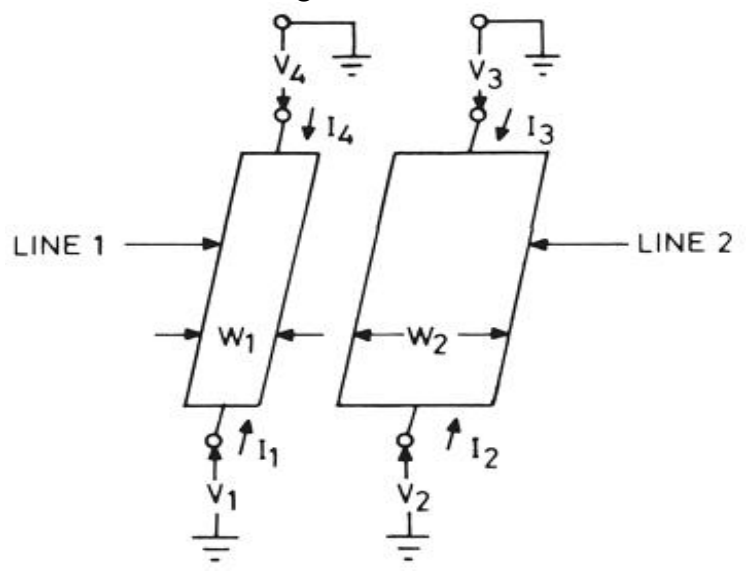

Figure 4: A pair of coupled lines with unequal impedances.

For coupled mode analysis, the voltage on one line is written in terms of the currents on both lines and the selfand mutual impedances. Similarly the current is written in terms of voltages and admittances. Eliminating currents or voltages yields the coupled equations. The solution of these coupled equations determines the propagation constants for the two modes [3].

Assuming a variation of the type $V(z)=V_{0} e^{-y z}$ for the voltages $v_{1}$ and $v_{2}$, the coupled equations resultants, reduce to the eigenvalue equation.

$$
\left[\gamma^{4}-\gamma^{2}\left(a_{1}+a_{2}\right)+a_{1} a_{2}-b_{1} b_{2}\right] v_{0}
$$


Where

$$
\begin{aligned}
& a_{1}=Z_{1} Y_{1}+Z_{m} Y_{m} \\
& b_{1}=Z_{1} Y_{m}+Z_{m} Y_{2} \\
& a_{2}=Z_{2} Y_{2}+Z_{m} Y_{m} \\
& b_{2}=Z_{2} Y_{m}+Z_{m} Y_{1}
\end{aligned}
$$

Where $Z_{j}$, and $Y_{j}(j=1,2)$ are self-impedances and selfadmittances per unit length of lines 1 and 2 and $Z_{m}$ and $Y_{m}$ are mutual impedance and mutual admittance per unit length, respectively. A time variation $\mathrm{e}^{\mathrm{jwt}}$ is assumed.

The solution of equation (4) leads to the following four roots for $\mathrm{g}$ :

$$
\gamma_{c, \pi}^{2}=\frac{a_{1}+a_{2}}{2} \mp \frac{1}{2}\left[\left(a_{1}+a_{2}\right)^{2}+4 b_{1} b_{2}\right]^{\frac{1}{2}}
$$

The subscripts "c" and " $p$ " refer to the $\mathrm{c}$ and $\mathrm{p}$ modes for asymmetric coupled lines. The propagation constants for these modes, $\gamma \mathrm{c}$ and $\gamma \mathrm{p}$, correspond to in-phase and antiphase waves, which reduce to even- and odd-mode waves, respectively, for symmetrical lines.

As discussed above, asymmetric coupled lines can be represented in terms of two modes called $\mathrm{c}$ and $\mathrm{p}$ modes. The phase constants and the characteristic impedances of these modes are related to the line constants in the following manner [3]:

$\gamma_{c, \pi}=j \beta_{c, \pi}$

$$
\begin{aligned}
& =j \frac{\omega}{\sqrt{2}}\left[L_{1} C_{1}+L_{2} C_{2}\right. \\
& -2 L_{m} C_{m} \\
& \left. \pm \sqrt{\left(L_{2} C_{2}-L_{1} C_{1}\right)^{2}+4\left(L_{m} C_{1}-L_{2} C_{m}\right)\left(L_{m} C_{2}-L_{1} C_{m}\right)}\right]^{1 / 2} \\
& Z_{c 1}=\frac{\omega}{\beta_{c}}\left(L_{1}-\frac{L_{m}}{R_{\pi}}\right)=\frac{\beta_{c}}{\omega}\left(\frac{1}{C_{1}-R_{c} C_{m}}\right) \\
& Z_{\pi 1}=\frac{\omega}{\beta_{\pi}}\left(L_{1}-\frac{L_{m}}{R_{c}}\right)=\frac{\beta_{\pi}}{\omega}\left(\frac{1}{C_{1}-R_{\pi} C_{m}}\right)
\end{aligned}
$$

And

$R_{c, \pi}=$

$\frac{L_{2} C_{2}-L_{1} C_{1} \pm \sqrt{\left(L_{2} C_{2}-L_{1} C_{1}\right)^{2}+4\left(L_{m} C_{2}-L_{1} C_{m}\right)\left(L_{m} C_{1}-L_{2} C_{m}\right)}}{2\left(L_{m} C_{2}-L_{1} C_{m}\right)}$

Self-capacitances $\mathrm{C}_{1}$ and $\mathrm{C}_{2}$, self-inductances $\mathrm{L}_{1}$ and $\mathrm{L}_{2}$ as well as mutual capacitance $\mathrm{C}_{\mathrm{m}}$ and mutual inductance $\mathrm{Lm}$ can be determined from quasi-static analysis

It was pointed out earlier that the $\mathrm{c}$ and $\mathrm{p}$ modes reduce to the even and odd modes, respectively, for symmetric coupled lines. The propagation constants for these modes are given by

$$
\gamma_{e, o}=\gamma_{c, \pi}=\left[\left(Y_{o} \pm Y_{m}\right)\left(Z_{o} \pm Z_{m}\right)\right]^{\frac{1}{2}}
$$

Where

$$
Y_{o}=Y_{1} \text { or } Y_{2} \quad Z_{o}=Z_{1} \text { or } Z_{1}
$$

And the voltage ratio is simplified:

$$
R_{c}=\frac{V_{2}}{V_{1}}=+1 \quad(\text { for the even mode })
$$

$$
R_{\pi}=\frac{V_{2}}{V_{1}}=-1 \quad(\text { for the odd mode })
$$

In terms of line constants, the characteristics of even and odd modes can be obtained from (7) to (9) by substituting $\mathrm{L}_{1}$ $=\mathrm{L}_{2}=\mathrm{L}_{\mathrm{o}}$ and $\mathrm{C}_{1}=\mathrm{C}_{2}=\mathrm{C}_{\mathrm{o}}$. The expressions are given as

$\beta_{e, o}=\omega\left[L_{o} C_{o}-L_{m} C_{m} \pm\left(L_{m} C_{o}-L_{o} C_{m}\right)\right]^{1 / 2}$

$Z_{o e}=\frac{\omega}{\beta_{e}}\left(L_{o}+L_{m}\right)=\frac{\beta_{e}}{\omega}\left(\frac{1}{C_{0}-C_{m}}\right)$

And

$$
\begin{gathered}
Y_{o e}=\frac{1}{Z_{o e}} \\
Z_{o o}=\frac{\omega}{\beta_{o}}\left(L_{o}-L_{m}\right)=\frac{\beta_{o}}{\omega}\left(\frac{1}{C_{o}+C_{m}}\right)
\end{gathered}
$$

And

$$
Y_{o o}=\frac{1}{z_{o o}}
$$

Where $\beta \mathrm{e}, \mathrm{o}$ are the phase constants of lossless coupled lines given by $\gamma \mathrm{e}, \mathrm{o}=\mathrm{j} \beta \mathrm{e}, \mathrm{o}$. The lines are characterized by inductance per unit length $\mathrm{L}_{o}$ and capacitance per unit length $\mathrm{C}_{0}$. The mutual inductance is $\mathrm{L}_{\mathrm{m}}$ and the mutual capacitance is $\mathrm{C}_{\mathrm{m}}$.

\section{Wavelet Theory and Method of Moments}

Researchers are now faced with an ever increasing variety of wavelet bases to choose from. While the choice of the "best" wavelet is obviously application dependent, it can be useful to isolate a number of properties and features that are of general interest to the user. Since wavelets may have some nice properties, including symmetry, vanishing moments, compact support and orthogonality, the construction of a wavelet has become a very popular scientific research subject, especially in mathematics and engineering [13]. One can construct wavelets $\psi$ such that the dilated and translated family is an orthonormal basis of $\mathrm{L}^{2}(\mathrm{R})$.

$$
\left\{\psi_{j, n}(t)=\frac{1}{\sqrt{2^{j}}} \psi\left(\frac{t-2^{j} n}{2^{j}}\right)\right\}_{(j, n) \in Z^{2}}
$$

Orthogonal wavelets dilated by $2^{\mathrm{j}}$ carry signal variations at the resolution $2^{\mathrm{j}}$. The construction of these bases can be related to multiresolution signal approximations. Most applications of wavelet bases exploit their ability to efficiently approximate particular classes of functions with few nonzero wavelet coefficients.

\subsection{Wavelets Bases}

\subsubsection{Daubechies Wavelets}

The dbN wavelets are the Daubechies extremal phase wavelets. $\mathrm{N}$ refers to the number of vanishing moments. In the construction of Daubechies wavelets, we seek a finite set of nonzero coefficients $h_{k}$ in the dilation equation [4] 


$$
\varphi(t)=\sum_{k} h_{k} \sqrt{2} \varphi(2 t-k)
$$

Recalling that

$$
\hat{h}\left(\frac{\omega}{2}\right)=\sum_{k} \frac{h_{k}}{\sqrt{2}} e^{-i\left(\frac{\omega}{2}\right) k}
$$

In general, for Daubechies scalets $\mathrm{hn}=0$ for $\mathrm{n}<0$ and $\mathrm{n}>$ $2 \mathrm{~N}+1$, the support $\phi=[0,2 \mathrm{~N}-1]$, and the support $\psi=[1-$ $\mathrm{N}, \mathrm{N}]$, where $\mathrm{N}$ is the order, or the number of vanishing moments. Figure 5 displays the graphs $\psi$ for $\mathrm{N}=4$.

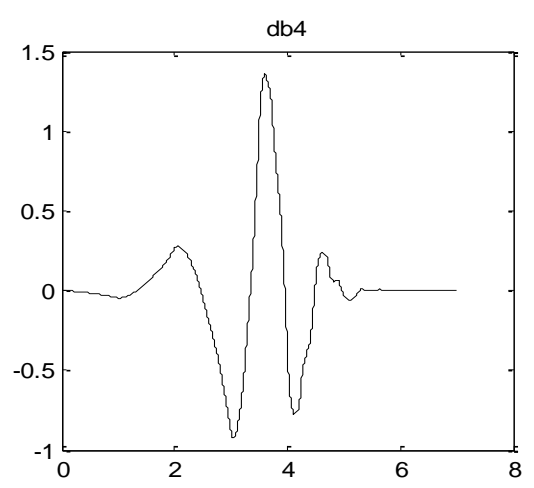

Figure 5: Daubechies Wavelets

\subsubsection{Symmlets}

Daubechies wavelets are very asymmetric. To obtain a symmetric or anti-symmetric wavelet, the filter $\mathrm{h}$ must be symmetric or anti-symmetric with respect to the center of its support, which means that $\widehat{h}(\omega)$ has a linear complex phase. The Daubechies Symmlet filters are obtained by optimizing the choice of the square $\operatorname{root} R\left(\mathrm{e}^{-\mathrm{i} \omega}\right)$ of $\mathrm{Q}\left(\mathrm{e}^{-\mathrm{i} \omega}\right)$ to obtain an almost linear phase. The resulting wavelets still have a minimum support $[-p+1, p]$ with $p$ vanishing moments, but they are more symmetric, as illustrated by Figure 6 for $\mathrm{N}=4$ [6-13].

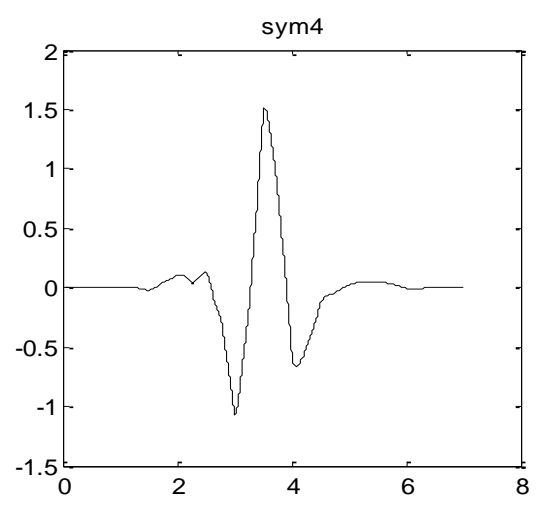

Figure 6: Symmlets wavelets

\subsubsection{Biorthogonal Wavelet}

We study the design of biorthogonal wavelets with a minimum-size support for a specified number of vanishing moments. Symmetric or anti-symmetric compactly supported spline biorthogonal wavelet bases are constructed with a technique introduced in [1]. The resulting Spline biorthogonal wavelets for $(p=4, \hat{p}=4)$ is illustrated in Fig. 7 [13].

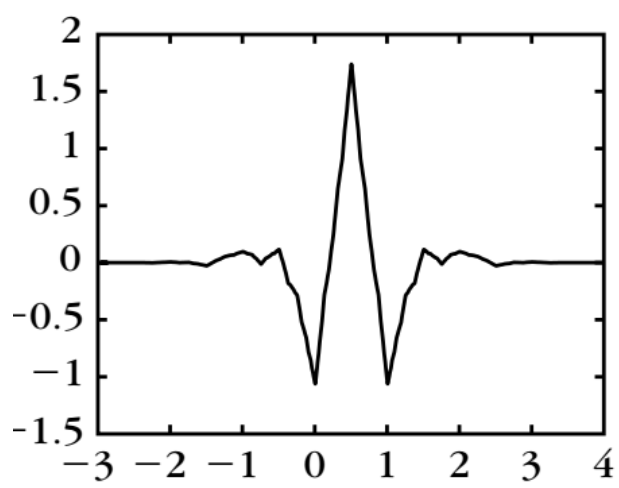

Figure 7: Biorthogonal wavelet [13]

\subsubsection{Coiflets Wavelets}

An orthonormal wavelet system with compact support is called the Coifman wavelet system of order $\mathrm{L}$ if $\phi(\mathrm{t})$ and $\psi(\mathrm{t})$ have $\mathrm{L}-1$ and $\mathrm{L}$ vanishing moments, respectively [4],

$$
\begin{array}{ll}
\int d t t^{l} \psi(t)=0 & l=0,1,2, \ldots, L-1 \\
\int d t t^{l} \varphi(t)=0 & l=0,1,2, \ldots, L-1
\end{array}
$$

The unique property of the Coiflets is contained in (20), namely the vanishing moments of the scalets. This property can be shown to yield

$$
\sum_{k} k h_{k}=0
$$

The resultant Coiflets scalet and wavelet of order $L=4$ are plotted along with their Fourier transform (in magnitude) in Fig. 8.

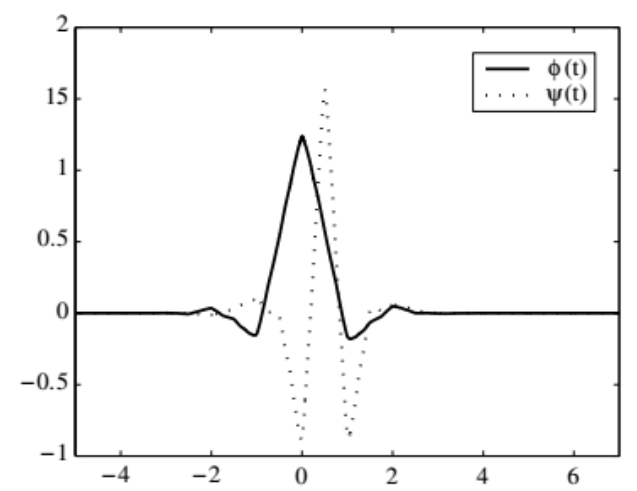

Figure 8: Coiflets scalet $\phi$ and wavelet $\psi(\mathrm{L}=4)$.

\subsection{Method of Moments and wavelets}

It is easier to expand a given function in a wavelet basis than to expand an unknown function in wavelets while solving the corresponding integral equation by the MoM $[5,14]$. 
The wavelets are applied directly upon the integral equation. The density of charge will be represented as a linear combination of the set wavelet functions and scaling functions as fellow $[10,15]$ :

$$
\rho(x 0)=\sum_{n} a_{n} \emptyset_{j . n}(x 0)+\sum_{m=j}^{2^{j}-1} \sum_{n} C_{m . n} \psi_{m . n}(x 0)
$$

The main mathematical properties which enable sparse matrix generation are the orthogonally and the vanishing moment. A function $\psi(\mathrm{x})$ is said to have vanished moment of $\mathrm{N}$ order if:

$$
\int_{-\infty}^{+\infty} \mathrm{x}^{\mathrm{n}} \psi(\mathrm{x}) \mathrm{dx}=0 \quad \forall n=0,1 \ldots .(N-1)
$$

When applying equation 22 into equation 1 we obtain:

$V(x)$

$=\int_{(a-w) / 2}^{(a+w) / 2}\left[\sum_{\mathrm{n} \in \mathrm{Z}} \mathrm{a}_{\mathrm{n}} \emptyset_{\mathrm{j} . \mathrm{n}}(x 0)\right.$
$\left.+\sum_{\mathrm{m}=\mathrm{j}}^{2^{\mathrm{j}}-1} \sum_{\mathrm{l}} \mathrm{C}_{\mathrm{m} . \mathrm{l}} \Psi_{\mathrm{m} . \mathrm{l}}(x 0)\right][G(x, x 0)] d x 0$

Applying testing functions we obtain:

$$
\begin{aligned}
& \left\langle V(x), \sum_{\mathrm{n} \in \mathrm{Z}} \emptyset_{\mathrm{j} . \mathrm{n}}(x)+\sum_{\mathrm{m}=\mathrm{j}}^{2^{\mathrm{j}}-1} \sum_{\mathrm{l}} \Psi_{\mathrm{m} . \mathrm{l}}(x)\right\rangle \\
& =\int_{(a-w) / 2}^{(a+w) / 2}\left\langle\left[\sum_{\mathrm{n} \in \mathrm{Z}} \mathrm{a}_{\mathrm{n}} \emptyset_{\mathrm{j} . \mathrm{n}}(x 0)\right.\right. \\
& \left.+\sum_{\mathrm{m}=\mathrm{j}}^{2^{\mathrm{j}}-1} \sum_{\mathrm{l}} \mathrm{C}_{\mathrm{m} . \mathrm{l}} \psi_{\mathrm{m} . \mathrm{l}}(x 0)\right][G(x, x 0)], \sum_{\mathrm{n} \in \mathrm{Z}} \emptyset_{\mathrm{j} . \mathrm{n}}(x) \\
& \left.+\sum_{\mathrm{m}=\mathrm{j}}^{2^{\mathrm{j}}-1} \sum_{\mathrm{l}} \Psi_{\mathrm{m} . \mathrm{l}}(x)\right\rangle d x 0
\end{aligned}
$$

This set of equations can be written in matrix form:

$$
\left[\begin{array}{cc}
{\left[\mathrm{Z}_{\emptyset, \emptyset}\right]} & {\left[\mathrm{Z}_{\emptyset, \psi}\right]} \\
{\left[\mathrm{Z}_{\psi, \varnothing}\right]} & {\left[\mathrm{Z}_{\psi, \psi}\right]}
\end{array}\right]\left[\begin{array}{c}
\mathrm{a}_{\mathrm{n}} \\
\mathrm{c}_{\mathrm{m}, \mathrm{n}}
\end{array}\right]=\left[\begin{array}{c}
<\mathrm{V}, \emptyset_{\mathrm{j}, \mathrm{n}}> \\
<\mathrm{V}, \Psi_{\mathrm{m}, \mathrm{n}}>
\end{array}\right]
$$

Where:

$$
\begin{aligned}
& {\left[Z_{\emptyset, \emptyset}\right]=<\emptyset_{j, n}, \int_{\frac{a-w}{2}}^{\frac{a+w}{2}} \emptyset_{j, n} G(x, x 0) d x 0>} \\
& {\left[Z_{\varnothing, \psi}\right]=<\emptyset_{j, n}, \int_{\frac{a-w}{2}}^{\frac{a+w}{2}} \psi_{m, n} G(x, x 0) d x 0>} \\
& {\left[Z_{\psi, \varnothing}\right]=<\psi_{m, n}, \int_{\frac{a-w}{2}}^{\frac{a+w}{2}} \emptyset_{j, n} G(x, x 0) d x 0>} \\
& {\left[Z_{\psi, \psi}\right]=<\psi_{m, n}, \int_{\frac{a-w}{2}}^{\frac{a+w}{2}} \psi_{m, n} G(x, x 0) d x 0>}
\end{aligned}
$$

Matrix $Z_{\psi, \psi}$ is very sparse because both of the expansion and testing functions are wavelets. Matrices $\mathrm{Z}_{\emptyset, \Psi}$ and $\mathrm{Z}_{\Psi, \varnothing}$ are composed of a mix of scalet and wavelet. Matrix $Z_{\emptyset, \emptyset}$ is dense because both of the expansion and testing functions are scalets. These submatrices represent the interaction between the sources and fields in different subspaces.

\section{Results and discussions}

In this section the example is presented to verify the validity of the proposed new basis functions in this paper. Consider a two coupled symmetric microstrip (figure 3) with $\mathrm{w} 1=\mathrm{w} 2=0.635, \mathrm{~W} / \mathrm{h}=0.005$ to 1.5 , and $\mathrm{s}=2 \mathrm{~mm}$. The substrate thickness is $\mathrm{h}=0.635 \mathrm{~mm}$ and the relative permittivity of the substrate is $\varepsilon r=9.6$ [3]. We concentrate on the line constants, the characteristics of even and odd and electric coupling, as this quantity depends strongly on the accuracy of the results.

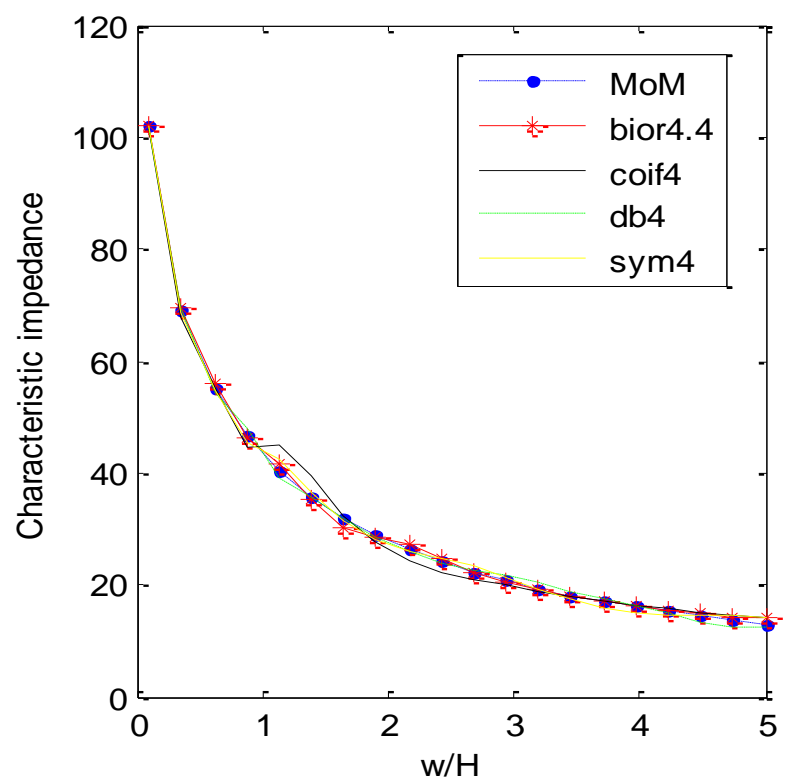

(a) 


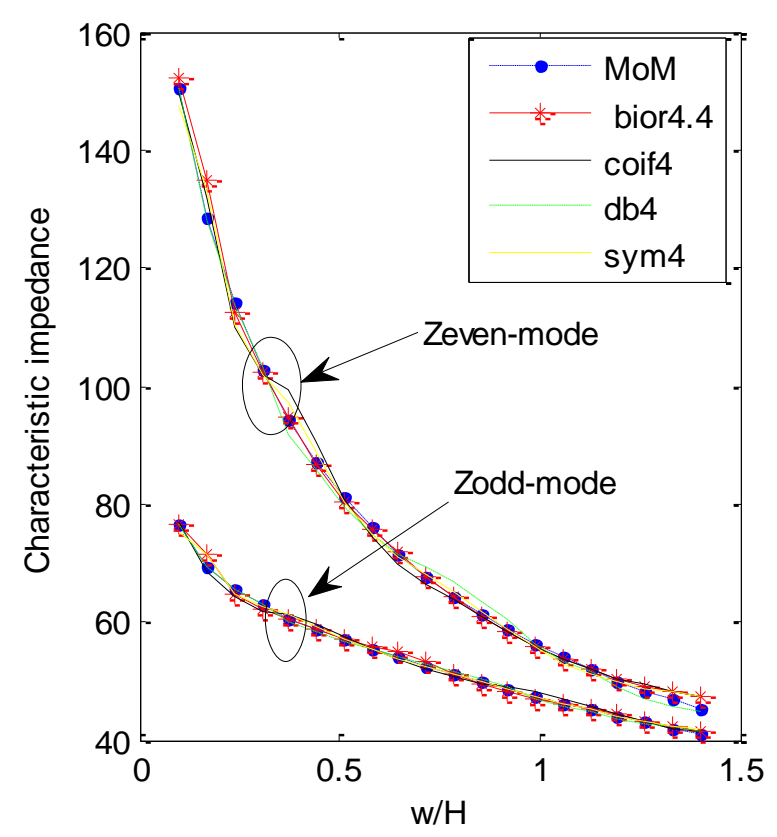

(b)

Fig. 9: Characteristic impedance of microstrip line (a) and Even- and odd-mode characteristic impedances for coupled microstrip lines (b).

Fig. 9 (a) and Fig. 9 (b) respectively show the characteristic impedance of the shielded microstrip and the even- and oddmode characteristic impedances for coupled microstrip lines by the classical pulse functions basis as well as Biorthogonal, Coiflets, Daubechies, and Symlets wavelet bases with strip width $\mathrm{W} / \mathrm{h}$. We observe that the characteristic impedance decreases when the ratio $\mathrm{W} / \mathrm{h}$ increases. This variation is due to the microstrip line width, $\mathrm{w}$, and when $\mathrm{w}$ increases, the capacity of the line increases. We can see a good agreement between the two methods, especially for the Biorthogonal and Symlets wavelets. These results agree well with the previous work published in [3], [1] and [10].

Table 1 summarizes the numerical results of all aforementioned wavelet bases in terms of the average percentage sparsity achieved in the IM, the CPU Time to reverse IM, and the relative error. The calculations are made on a PC computer with AMD Dual-Core $1.30 \mathrm{GHz}$ CPU, 2 GB memory and Windows 7 Professional system.

Table 1: Execution times, sparsity and relative error versus

\begin{tabular}{|c|c|c|c|c|c|}
\hline & MoM & Db4 & Sym4 & Coif4 & Bior4.4 \\
\hline Sparsity \% & & 68.57 & 71.42 & 52.32 & 69.44 \\
\hline $\begin{array}{l}\text { CPU Time } \\
\text { to reverse } \\
\text { IM }(\mathrm{ms})\end{array}$ & 9.168 & 3.346 & 1.672 & 8.133 & 2.641 \\
\hline $\begin{array}{l}\text { CPU Time } \\
\text { reduction \% }\end{array}$ & & 63.50 & 81.762 & 11.289 & 71.193 \\
\hline $\begin{array}{l}\text { Relative } \\
\text { error }\end{array}$ & & 0.0011 & 0.0003 & 0.0018 & 0.0015 \\
\hline
\end{tabular}

As seen in Table 1, the basis wavelets expansion extracts the variation of characteristic impedance more rapidly than the conventional MoM. Table 1 also reveals that Biorthogonal and Symlets wavelets extract the variation of characteristic impedance more rapidly than the Coiflets, and Daubechies. In particular, the Symlets (CPU Time reduction $=81.762 \%$ and Relative error $=0.0003$ ) appears to be the most appropriate choice for analyzing the coupled microstrip line.

This is sought to be due to its regularity and compact support. The regularity of wavelet has mostly a cosmetic influence on the error introduced by thresholding or quantizing the wavelet coefficients.

Fig. 10 shows the sparsity pattern of the most illconditioned IM for different wavelets, db4: Daubechies wavelet, sym4: Symlets wavelet, coif4: Coiflets wavelet, and bior4: Biorthogonal wavelet. By means of Fig. 10, priori locations of non-significant matrix elements have been estimated, and evaluation of small elements at impedance matrix has been avoided.
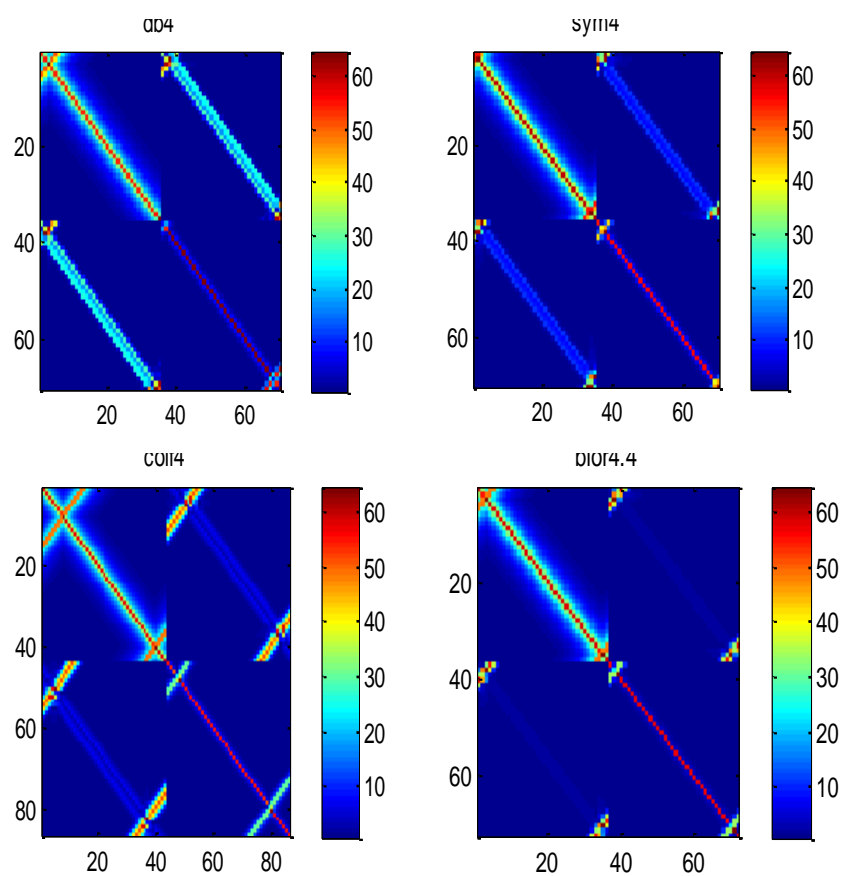

Figure 10: Gray-image of a typical coefficient matrix.

The coupling coefficient is given by the following formula [16]:

$$
k=\frac{Z_{o e}-Z_{o o}}{Z_{o e}+Z_{o o}} \quad \text { with } 0<K<1
$$

Figure 11 shows the variations of the coupling coefficient as a function of the gap $\mathrm{S}$ between the two lines of coupled microstrip line, for different dielectric constants of substrate. 


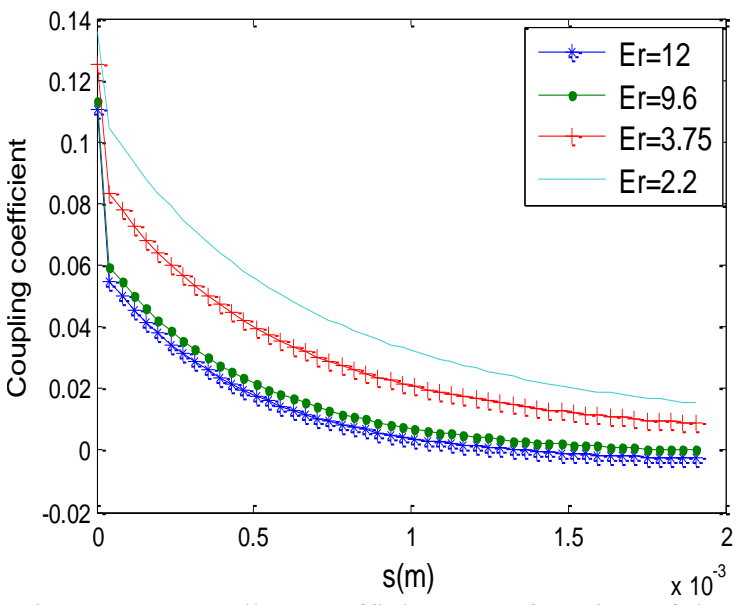

Figure 11: Coupling coefficient as a function of the gap $s$.

We observe also the electric coupling $\mathrm{k}$ a depend of dielectric constant. The lower the dielectric constant, the stronger is the electric coupling. The numerical results also indicate that for low values of dielectric constant the variation of electric coupling with $\mathrm{gr}$, is rapid while it is rather slow for high values of $\varepsilon r$. These results agree well with the previous work published in [17].

\section{Conclusions}

In this paper, we studied the several of wavelets in the goal to evaluate the characteristic impedance of the shielded microstrip and the even- and odd-mode characteristic impedances for coupled microstrip lines. We have presented the numerical results of the even- and odd-mode characteristic impedances, coupling coefficients, average percentage sparsity achieved in the IM, the CPU Time to reverse IM, and the relative error obtained using methods of moments-wavelets. The MoM-wavelets of the four types of wavelets, namely the Daubechies, Coiflets, Biorthogonal and Symlets have been investigated. The comparison between MoM and several wavelets provides a good agreement. This new basis possesses the properties of rapid convergence. Taking full advantages of wavelets analysis, it has been demonstrated that highly sparse matrice is obtained. Thus the calculation time is significantly reduced. In particular, the Symlets wavelets (Sparsity $=71.42 \%$, CPU Time reduction $=81.762 \%$ and Relative error $=0.0003$ ) appears to be the most appropriate choice of analyzing the coupled microstrip line.

\section{References}

[1] A.M. Abbosh, Analytical closed-form solutions for different configurations of parallel-coupled microstrip lines, IET Microw. Antennas Propag., Vol. 3, Iss. 1, pp. 137-147, 2009.

[2] L. Deias , M. Lai, G. Mazzarella, Analysis of Microstrip Coupled Lines over a Perfect Magnetic Conductor, Antennas and Propagation Conference LAPC, Loughborough, , UK, pp.1-3, 2011.
[3] Ramesh Garg, Inder Bahl, and Maurizio Bozzi, Microstrip Lines and Slotlines, Third Edition, Artech House, Boston and London, 2013.

[4] Georgew. Pan, Wavelets in Electromagnetics and Device Modeling, John Wiley \& Sons, Inc, Hoboken, New Jersey, 2003.

[5] E. Francomanoa, A. Tortorici, and al., Wavelet-like bases for thin-wire Integral equations in electromagnetics, Journal of Computational and Applied Mathematics, Vol. 175, pp. 77-86, 2004.

[6] Jaideva C. Goswami, and Andrew K. Chan, Fundamentals Wavelets Theory, Algorithms, and Applications, John Wiley \& Sons, Hoboken, New Jersey, 2011.

[7] Nassima Tidjani, and al., Electromagnetic Coupling Modeling of Microstrip Lines Used in Switching Power Supplies, Journal of Control Science and Engineering, Vol.1, pp. 55-59, 2013.

[8] Jen-Tsai Kuo, Wei-Hsiu Hsu, and Al., Parallel Coupled Microstrip Filters with Suppression of Harmonic Response, Ieee Microwave And Wireless Components Letters, Vol. 12, NO. 10, pp. $383-385,2002$.

[9] Nejla Oueslat and Taoufik Aguili, New Implementation of the Moment Method Based on the Impedance Operator to Study the Dispersion Characteristics of Microstrip Lines, International Journal of Computer Science Issues, Vol. 11, Issue 5, pp.59-67, 2014.

[10] Mohamed Bayjja, Mohamed Boussouis, and Naima Amar Touhami, Studying the Influence of the Number Vanishing Moments of Daubechies Wavelets for the Analysis of Microstrip Lines, Progress In Electromagnetics Research Letters, Vol. 62, pp. 57-64, 2016.

[11] Ikmo Park, Raj Mittra, and M. I. Aksun, Numerically Efficient Analysis of Planar Microstrip Configurations Using Closed-Form Green's Functions, IEEE Transactions On Microwave Theory And Techniques, Vol. 43, NO. 2, pp. 394- 400, 1995.

[12] P. Silvester, B.S., M.A.Sc, Ph.D., TEM wave properties of microstrip transmission lines, PROC. IEE, Vol. 115, No. 1, pp. 43 - 48, 1968.

[13] Stéphane Mallat, A Wavelet Tour of Signal Processing The Sparse Way, Elsevier Inc, Newyork, Oxford, Paris , 2009.

[14] Mohamed Bayjja, and al., Orthogonal and Biorthogonal Compactly Supported Wavelets in Modeling the Circular Loop Antenna, International Journal of Microwave and Optical Technology, VOL.12, NO.5, pp. 331-337, 2017.

[15] Walton C. Gibson, The Method of Moments in Electromagnetics, Second Edition, Taylor \& Francis, New York, 2015.

[16] Wadell Brian C, Transmission line design handbook, Artech House, Boston, 1991. 
[17] Jia-Sheng Hong, M.J. Lancaster and Al., Couplings of Microstrip Square Open-Loop Resonators, IEEE Tran. on MTT, Vol. 44, No. 12, pp. 2099 - 2109, 1996. 